\title{
Extragenital testing increases case detection of gonorrhea and chlamydia: The impact of implementing nucleic acid amplification testing
}

\author{
Dara Spatz Friedman ${ }^{1,2}$, Patrick O’Byrne ${ }^{1,2 \star}$
}

\begin{abstract}
Background: Nucleic acid amplification testing (NAAT) was validated in Ontario in 2018 to test for chlamydia and gonorrhea at extragenital (pharyngeal, rectal) sites. Prior to this validation, extragenital testing could be done only by culture in Ontario. The objective of this study was to determine the number and proportion of gonorrhea and chlamydia cases that were detected exclusively through extragenital (pharyngeal and/or rectal) testing after the implementation of extragenital NAAT for these two infections at Sexual Health Clinic among gay, bisexual, and other men who have sex with men (gbMSM).
\end{abstract}

Methods: Case and laboratory data from before and after NAAT implementation were used to compare the rates of diagnosis of gonorrhea and chlamydia among gbMSM who presented at Sexual Health Clinics and the percent increase in diagnoses in GbMSM in the entire population.

Results: Among gbMSM seen at the clinic after implementation of NAAT testing, $70 \%$ of gonorrhea cases and $65 \%$ of chlamydia cases were detected exclusively at extragenital sites, corresponding to a four and two-fold increase, respectively, in the average annual number of cases diagnosed. As well, although approximately $50 \%$ more pharyngeal than rectal testing occurred, a higher proportion of chlamydia cases were detected rectally than would have been expected; this was not the case for gonorrhea, where most infections were pharyngeal.

Conclusion: It is important that clinicians perform extragenital testing among gbMSM who have sexual contact involving extragenital sites with more than one partner.
This work is licensed under a Creative Commons Attribution 4.0 International License.

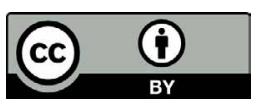

Affiliations

1 Ottawa Public Health, Ottawa, $\mathrm{ON}$

2 University of Ottawa, Ottawa, $\mathrm{ON}$

*Correspondence:

patrick.obyrne@uottawa.ca

Suggested citation: Friedman DS, O'Byrne P. Extragenital testing increases case detection of gonorrhea and chlamydia: The impact of implementing nucleic acid amplification testing. Can Commun Dis Rep

2020:46(9):285-91. https://doi.org/10.14745/ccdr.v46i09a06

Keywords: gonorrhea, chlamydia, NAAT, extragenital, pharynx, rectum

\section{Introduction}

Neisseria gonorrhoea (N. gonorrhoea) and Chlamydia trachomatis (C. trachomatis) are the most commonly reported sexually transmitted infections (STIs) in Canada, and their incidence is increasing (1). In Ottawa (Ontario), the observed incidence rates of gonorrhea and chlamydia have also been increasing over the last 20 years; however, the rate of increase for gonorrhea has been significantly higher since 2016

(Figure 1) (2). A total of 90 and 390 cases of gonorrhea and chlamydia, respectively, were diagnosed per 100,000 population in 2018 in Ottawa; up from 38 and 335 cases per 100,000 in 2016 (2). The rates of these infections in Ottawa have increased most markedly among gay, bisexual and men who have sex with men (gbMSM). In 2018, gbMSM comprised $45 \%$ of gonorrhea and $10 \%$ of chlamydia cases (2).

While gonorrhea and chlamydia are classically considered infections of the genital mucosa, infection of extragenital sites, such as the pharynx and rectum, is common (3). Because these infections are often asymptomatic or clinically non-specific when symptomatic, they are likely a source of ongoing transmission (4). For the same reasons, diagnosis of these infections requires laboratory confirmation (5). Culture was the primary detection method for urogenital, rectal and pharyngeal infections until the availability of more sensitive molecular testing, such as 
Figure 1: Number and rate of diagnosis of gonorrhea and chlamydia, Ottawa, 1999-2018

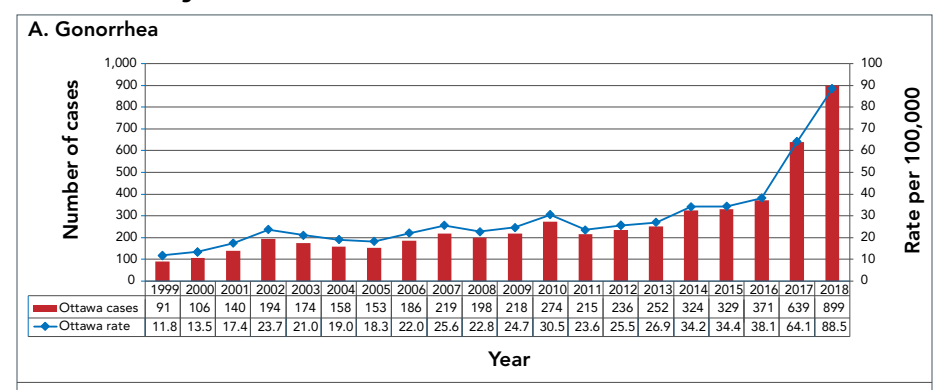

B. Chlamydia

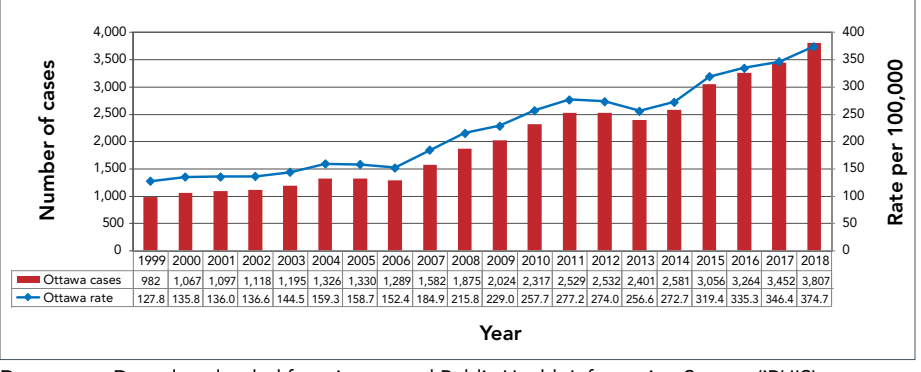

Data notes: Data downloaded from integrated Public Health Information System (iPHIS), November 8, 2019 by Ottawa Public Health (OPH). 2019 count includes reports through September 30, 2019; the 2019 rate is adjusted for partial year

nucleic acid amplification testing (NAAT) (6). Although Canadian Guidelines on Sexually Transmitted Infections (5) recommend NAAT, it is not approved by Health Canada for extragenital testing, unless validated by local laboratories.

In Ontario, extragenital NAAT was validated at STI clinics in Ottawa and Toronto among gbMSM, sex trade workers and their clients, and known contacts of persons diagnosed with gonorrhea or chlamydia (7). This validation involved clinicians performing extragenital cultures and NAAT for the foregoing clients when they consented to such testing. All such samples were submitted to the Public Health Ontario Laboratory for testing and comparison. NAAT, compared with culture, had 92.2\% sensitivity and $99.9 \%$ specificity for pharyngeal testing and $99.4 \%$ sensitivity and $99.9 \%$ specificity for rectal testing. Gonorrhea NAAT, compared with culture, showed similar results; identifying $100 \%$ sensitivity for both pharyngeal and rectal testing and $98.2 \%$ and $99.0 \%$ specificity for pharyngeal and rectal specificity, respectively (7). Per site, culture, compared with NAAT, had a detection rate of $13 \%$ for pharyngeal gonorrhea, $67 \%$ for rectal gonorrhea, $17 \%$ for pharyngeal chlamydia and $38 \%$ for rectal chlamydia. This validation of extragenital NAAT occurred between July and November 2017, followed by full implementation in April 2018. Despite this change in testing methodology, there were no clinical practice changes during this time. National guidelines recommended extragenital testing, and in the Sexual Health Clinic, such testing was routinely performed (using culture) when clinically indicated and was accepted by clients. Extragenital testing was carried out by clinicians who followed established clinical practices for collecting samples from these sites. Samples were then submitted to the Public Health
Ontario Laboratory. During the study period, patient-collected extragenital testing was not available.

While the potential impact of changing from culture to NAAT for the detection of extragenital chlamydia and gonorrhea in gbMSM patients has been established (8), the effect on surveillance at the population level has not been described. Furthermore, data on this topic have arisen from studies validating extragenital testing in STI clinics (8) and not from routine clinical practice that incorporates NAAT, such as that which occurs at the Sexual Health Clinic, where there are approximately 20,000 unique patient encounters per year. As such, in this paper, we enumerate extragenital gonorrhea and chlamydia case-finding pre/post-implementation of rectal and pharyngeal NAAT and show the effect of this testing on public health surveillance for these infections in Ottawa. Our analysis shows the proportion of gonorrhea and chlamydia infections that would be missed in an STI clinic if urogenital testing (but not NAAT) was performed.

\section{Methods}

Information about individual gonorrhea and chlamydia cases diagnosed among Ottawa residents from 1999 to 2019 was extracted from the integrated Public Health Information System (2) by Ottawa Public Health on November 19, 2019. Date of diagnosis, body site(s) testing positive, testing provider and gbMSM status were extracted and analysed for two time periods relative to validation and implementation of extragenital NAAT: pre-validation/pre-implementation (July 1, 2012-June 30, 2017) and implementation (May 1, 2018-October 31, 2019). Cases diagnosed through extragenital testing were those for whom extragenital testing was positive and genital testing was negative or not performed (Table 1). Cases diagnosed through genital testing were those for whom urogenital testing was positive regardless of whether extragenital testing was carried out and irrespective of the results of such extragenital testing.

\section{Table 1: Classification of testing by site of positive test result}

\begin{tabular}{|l|l|}
\multicolumn{1}{|c|}{ Category } & \multicolumn{1}{c|}{ Site of positive test result } \\
\hline \multirow{4}{*}{ Extragenital } & Pharyngeal only \\
\cline { 2 - 2 } & Rectal only \\
\cline { 2 - 2 } & Pharyngeal and rectal \\
\hline \multirow{4}{*}{ Genital } & Urogenital only \\
\cline { 2 - 2 } & Urogenital and pharyngeal \\
\cline { 2 - 2 } & Urogenital and rectal \\
\cline { 2 - 2 } & Urogenital and pharyngeal and rectal \\
\hline
\end{tabular}

The impact of extragenital testing on case-finding was evaluated in two ways using Stata v.16.0. First, the average annual number of cases of gonorrhea and of chlamydia diagnosed during each time period through extragenital or through genital testing of 
gbMSM presenting at the Sexual Health Clinic were calculated and compared using tests of proportion. Second, the percent increase in diagnoses in the entire population due to extragenital testing, as opposed to genital testing, was calculated for each infection and time period. Additionally, we performed a test of proportions to compare the percent positivity of pharyngeal and rectal gonorrhea and chlamydia infections, based on the total volume of such tests that were submitted for testing.

The percent positivity for extragenital NAAT conducted at the Sexual Health Clinic during a subset of the implementation period (April 9-August 8, 2019) was provided by the Public Health Ontario Laboratory, which tests all specimens from the Sexual Health Clinic.

\section{Results}

During the five-year period before the validation of extragenital NAAT (July 1, 2012-June 30, 2017), an average of 52 cases of gonorrhea and 83 cases of chlamydia were identified annually among gbMSM attending the Sexual Health Clinic (Table 2). In the 18 months (May 1, 2018-October 31, 2019) following implementation of extragenital NAAT at the Sexual Health Clinic, an average annual number of 220 and 210 cases of gonorrhea and chlamydia, respectively, were identified in gbMSM

(Table 3), and this was despite no increase in testing volume from 2015-2019.

Of the cases identified post-implementation, $70 \%$ of gonorrhea cases and $65 \%$ of chlamydia infections were identified from extragenital testing only; the remainder was identified from the testing of either exclusively genital or genital and extragenital

Table 2: Detection of gonorrhea or chlamydia by site of infection, gbMSM, Sexual Health Clinica, Ottawa, July 1, 2012-June 30, 2017

\begin{tabular}{|l|r|r|r|r|r|r|}
\hline & \multicolumn{3}{|c|}{ Gonorrhea } & \multicolumn{3}{c|}{ Chlamydia } \\
\cline { 2 - 8 } Cases & $\begin{array}{c}\text { Number } \\
\text { of cases }\end{array}$ & $\begin{array}{c}\text { Average } \\
\text { annual } \\
\text { number } \\
\text { of cases }\end{array}$ & $\begin{array}{c}\text { Percent } \\
\text { of all } \\
\text { cases }\end{array}$ & $\begin{array}{r}\text { Number } \\
\text { of cases }\end{array}$ & $\begin{array}{c}\text { Average } \\
\text { annual } \\
\text { number } \\
\text { of cases }\end{array}$ & $\begin{array}{c}\text { Percent } \\
\text { of all } \\
\text { cases }\end{array}$ \\
\hline All cases & 258 & 51.6 & $100.0 \%$ & 414 & 82.8 & $100.0 \%$ \\
\hline $\begin{array}{l}\text { Total with } \\
\text { known site }\end{array}$ & 258 & 51.6 & $100.0 \%$ & 413 & 82.6 & $99.8 \%$ \\
\hline Genital only & 140 & 28 & $54.3 \%$ & 215 & 43 & $51.9 \%$ \\
\hline $\begin{array}{l}\text { Genital and } \\
\text { extragenital }\end{array}$ & 37 & 7.4 & $14.3 \%$ & 27 & 5.4 & $6.5 \%$ \\
\hline $\begin{array}{l}\text { Extragenital } \\
\text { only }\end{array}$ & 81 & 16.2 & $31.4 \%$ & 171 & 34.2 & $41.3 \%$ \\
\hline Pharyngeal & 14 & 2.8 & $5.4 \%$ & 18 & 3.6 & $4.3 \%$ \\
\hline Rectal & 61 & 12.2 & $23.6 \%$ & 142 & 28.4 & $34.3 \%$ \\
\hline $\begin{array}{l}\text { Pharyngeal } \\
\text { and rectal }\end{array}$ & 6 & 1.2 & $2.3 \%$ & 11 & 2.2 & $2.7 \%$ \\
\hline Other & 0 & 0 & $0.0 \%$ & 1 & 0.2 & $0.2 \%$ \\
\hline
\end{tabular}

Abbreviation: gbMSM, gay, bisexual, and other men who have sex with men

ata source: Ministry of Health and Long-term Care, integrated Public Health Information

System, extracted by Ottawa Public Health, November 19, 2019

Does not include one case where site was unknown
Table 3: Detection of gonorrhea or chlamydia by site of infection, gbMSM, Sexual Health Clinica, Ottawa, May 1, 2018-October 31, 2019

\begin{tabular}{|c|c|c|c|c|c|c|}
\hline \multirow[b]{2}{*}{ Cases } & \multicolumn{3}{|c|}{ Gonorrhea } & \multicolumn{3}{|c|}{ Chlamydia } \\
\hline & $\begin{array}{l}\text { Number } \\
\text { of cases }\end{array}$ & $\begin{array}{c}\text { Average } \\
\text { annual } \\
\text { number } \\
\text { of cases }\end{array}$ & $\begin{array}{c}\text { Percent } \\
\text { of all } \\
\text { cases }\end{array}$ & $\begin{array}{l}\text { Number } \\
\text { of cases }\end{array}$ & $\begin{array}{c}\text { Average } \\
\text { annual } \\
\text { number } \\
\text { of cases }\end{array}$ & $\begin{array}{c}\text { Percent } \\
\text { of all } \\
\text { cases }^{b}\end{array}$ \\
\hline All cases & 348 & 219.8 & $100.0 \%$ & 332 & 209.7 & $100.0 \%$ \\
\hline $\begin{array}{l}\text { Total with } \\
\text { known site }\end{array}$ & 348 & 219.8 & $100.0 \%$ & 330 & 208.4 & $99.4 \%$ \\
\hline Genital only & 41 & 25.9 & $11.8 \%$ & 75 & 47.4 & $22.6 \%$ \\
\hline $\begin{array}{l}\text { Genital and } \\
\text { extragenital }\end{array}$ & 64 & 40.4 & $18.4 \%$ & 40 & 25.3 & $12.0 \%$ \\
\hline $\begin{array}{l}\text { Extragenital } \\
\text { only }\end{array}$ & 243 & 153.5 & $69.8 \%$ & 215 & 135.8 & $64.8 \%$ \\
\hline Pharyngeal & 106 & 66.9 & $30.5 \%$ & 19 & 12.0 & $5.7 \%$ \\
\hline Rectal & 72 & 45.5 & $20.7 \%$ & 152 & 96.0 & $45.8 \%$ \\
\hline $\begin{array}{l}\text { Pharyngeal } \\
\text { and rectal }\end{array}$ & 65 & 41.1 & $18.7 \%$ & 44 & 27.8 & $13.3 \%$ \\
\hline Other & 0 & 0.0 & $0.0 \%$ & 2 & 1.3 & $0.6 \%$ \\
\hline
\end{tabular}

Abbreviation: gbMSM, gay, bisexual, and other men who have sex with men

a Data source: Ministry of Health and Long-term Care, integrated Public Health Information

System, extracted by Ottawa Public Health, November 19, 2019

${ }^{b}$ Does not include two cases where site was unknown

sites. In contrast, significantly fewer infections ( $31 \%$ of gonorrhea and $41 \%$ of chlamydia, $p<0.00001$ for each) identified before validation of extragenital NAAT were detected from extragenital testing only.

Gonorrhea and chlamydia were differentially detected in the pharynx and rectum of gbMSM clients at the Sexual Health Clinic. Approximately 50\% more pharyngeal than rectal NAATs were carried out, and the percent positivity of pharyngeal and rectal testing was $8.3 \%$ and $9.9 \%$, respectively, for gonorrhea, and $1.6 \%$ and $11.3 \%$, respectively, for chlamydia (Personal communication, Public Health Ontario. Impact of pharyngeal and rectal Chlamydia trachomatis and Neisseria gonorrhoeae NAAT, July 2, 2019). It follows that, for gonorrhea, more cases, in terms of number and proportion of diagnoses, were detected by pharyngeal testing (49\%), compared with rectal testing (39\%) (Table 3; pharyngeal+pharyngeal/rectal versus rectal+pharyngeal/rectal). In contrast, for chlamydia, while the volume of pharyngeal and of rectal testing would predict the identification of more pharyngeal than rectal cases, a higher proportion of cases were identified through rectal testing (59\%) than expected, compared to pharyngeal testing (19\%) $(p<0.0001)$.

Lastly, the number of cases identified at the population level in Ottawa increased as a result of extragenital NAAT. This increase was most striking for gonorrhea: between 1999 and 2016 , the rate of gonorrhea increased an average of $13 \%$ each year; between 2016 and 2018, when extragenital NAAT was validated and implemented, the average annual increase was $65 \%$. Before the validation and implementation of extragenital NAAT, infections detected from extragenital sites alone resulted in the identification of $9 \%$ and $2 \%$ more gonorrhea and 
chlamydia cases, respectively, than that identified by genital testing (Figure 2). In contrast, $46 \%$ and $7 \%$ more gonorrhea and chlamydia cases, respectively, were identified in the 18 months following implementation of extragenital NAAT than what was identified using genital testing.

Figure 2: Case-finding by genital or extragenital testing, Ottawa, July 2012-October 2019
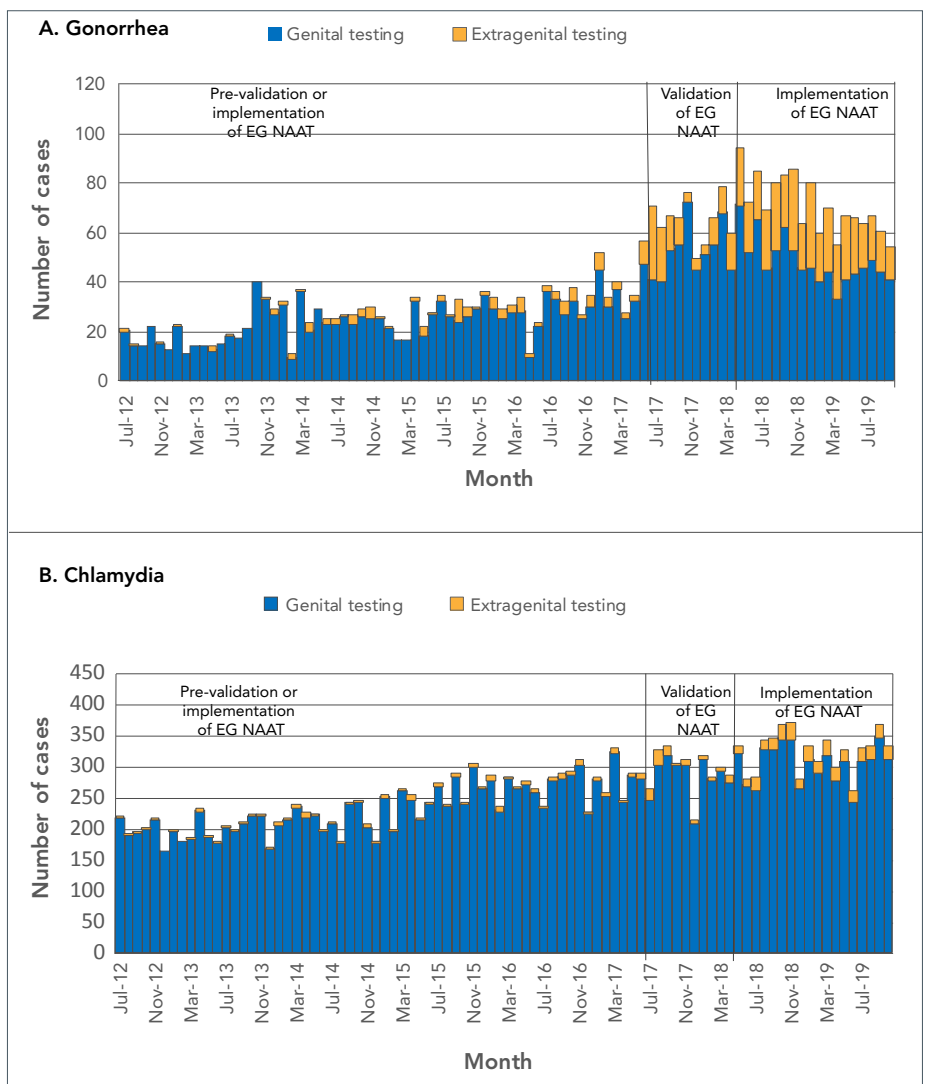

Abbreviation: EG NAAT, extragenital nucleic acid amplification testing

\section{Discussion}

Our analysis of gonorrhea and chlamydia testing at the Sexual Health Clinic, comparing the period when only culture was available for extragenital testing to the period when this testing was performed by NAAT for gbMSM and other identified groups, showed a significant increase in the proportion of these infections detected exclusively from extragenital sites. These findings indicate that a change in laboratory technology, without a change in clinical practice, resulted in an increase in case finding that had a profound effect on the number of diagnoses across Ottawa. For gonorrhea, the finding of extragenital infections among gbMSM visiting the Sexual Health Clinic increased from accounting for $31 \%$ of infections pre-NAAT to $70 \%$ post-NAAT; that is, under current practices, for every three cases where gonorrhea caused a genital infection, there were seven cases where this infection was exclusively extragenital. For chlamydia, the finding of extragenital infections increased from $41 \%$ pre-NAAT to $65 \%$ post-NAAT.

\section{Limitations}

These results must be interpreted considering three main limitations. First, the test results that we analyzed were from gbMSM who attended an STI clinic. The gbMSM seen by community providers might have had a lower prevalence of infection at extragenital sites. However, the observation that there were seven cases of extragenital gonorrhea for every three genital infections among this group of STI clinic clients suggests that many infections might be missed in the community, even if the underlying prevalence of infection was lower in the community. Conversely, it is also plausible that gbMSM who visit STI clinics could have a lower burden of STIs, as a result of routine health-seeking practices; meaning that higher rates of extragenital infection could be present among gbMSM who either seek testing less frequently or who do so from community providers. Second, because not all at-risk gbMSM in Ottawa might be receiving extragenital NAAT testing, these results could be an underestimate of its impact. That is, if all community providers performed extragenital NAAT testing on their at-risk gbMSM patients, the impact on detection might be even greater than documented here. Third, the increases seen in our analysis could be due to true increases in incidence, rather than missed infections that were identified by a new testing technology. That is, the increased rates of gonorrhea and chlamydia may have coincidentally corresponded with the change in testing technology. Comparisons with other jurisdictions could be made; however, these would be limited by the fact that currently reported epidemiologic data from these other locations do not differentiate by anatomic site of infection. Another strategy could have been to perform different analyses (e.g. time series approach), although this would require access to data not routinely available to STI clinic or public health unit staff and likely would not have identified markedly different findings, as the overall testing rates between 2015 through 2019 were relatively unchanged in our clinic.

\section{Recommendations}

Regarding clinical practice, the main recommendation stemming from our results is that providers should inform patients that they can acquire extragenital gonorrhea and chlamydia infections and should offer extragenital testing to gbMSM who engage in oral and/or anal sex with more than one sexual partner. Such screening should be offered irrespective of reported condom use, as studies $(7,9,10)$ have identified rectal infections despite patients' self-reported safe sexual practices. These results, moreover, support current Public Health Agency of Canada (5) STI screening guidelines to perform extragenital testing on at-risk patients, unless the patient declines testing or denies sexual contact at an extragenital site. These results also align with the United States Centers for Disease Control and Prevention (11) recommendation that extragenital screening should occur "regardless of condom use during exposure". Of issue, however, is that previous research has identified low rates of extragenital testing in many clinical settings $(12,13)$, which could result in many missed diagnoses. 
Importantly, our findings also align with Canadian HIV preexposure prophylaxis (PrEP) guidelines (14), which recommend performing STI testing every three months during follow-up. Providers who offer PrEP to gbMSM, but do not perform extragenital testing, may miss a sizeable number of infections. This is problematic from an HIV prevention perspective: because gonorrhea and chlamydia induce inflammation in the rectum, they can increase the risk of HIV acquisition (15). Identifying and treating rectal gonorrhea and chlamydia infections may, therefore, function not only as a control strategy for these two infections, but also as an HIV prevention intervention. Conversely, the detection of these infections is a clinical indication for PrEP, as they provide evidence of condomless receptive anal sex in the context of increased biologic risks for HIV acquisition $(16,17)$. Screening for these infections, therefore, also functions as an HIV prevention strategy, both to reduce biologic susceptibility and to identify persons in need of PrEP.

Because studies have shown that the acceptability of rectal swabs is lower than that of pharyngeal swabs $(18,19)$ providers might consider offering pharyngeal testing with or without rectal testing. While testing at both extragenital sites, when clinically indicated, is ideal, our analysis shows that pharyngeal testing alone would capture $70 \%$ and $29 \%$, respectively, of gonorrhea and chlamydia cases among gbMSM that would not be detected with urine testing alone. Testing the pharynx but not the rectum could also be appropriate, as more gbMSM report performing oral sex than receptive anal sex (20); in such cases, the addition of pharyngeal swabs would ensure more comprehensive testing. Offering pharyngeal swabs, irrespective of sexual orientation, may also provide a way for patients to agree to more comprehensive testing without having to disclose the sex of their sexual partners to healthcare providers. This might increase testing among gbMSM in primary care because up to $50 \%$ of gbMSM patients report being reluctant to disclose their sexual orientation to healthcare providers $(21,22)$. Offering pharyngeal swabs may also increase detection among other groups, although the proportion of infections at extragenital sites for non-gbMSM groups (e.g. males or females who have opposite sex partners) is unknown and warrants research. One possible downside to this recommendation is that our analysis identified more rectal than pharyngeal chlamydia infections, which aligns with a recent literature review that found rates of rectal chlamydia as $2.1 \%-23.0 \%$ (median $18.9 \%$ ) compared to $0 \%-3.6 \%$ (median 1.7\%) for pharyngeal infections (8). Although cellular tropism for columnar cells (which are found in the rectum but not the pharynx) may explain our findings, further research is required.

Lastly, our findings also yield recommendations for the interpretation of STI epidemiology. Indeed, the availability of a more sensitive laboratory test has changed our understanding of the epidemiology of gonorrhea and highlighted the need to review the site of gonorrhea infection when making conclusions about changes in STI rates. The observed incidence rates of gonorrhea and chlamydia in Ottawa have been increasing over the last 20 years. However, the observed rate of increase in gonorrhea has been significantly higher since validation of extragenital NAAT in 2017. The increase in case-finding due to the use of a new laboratory test suggests that, when this new laboratory method was not available, many existing infections went undiagnosed. Thus, the incidence of gonorrhea in the past might have been higher than previously thought, and the number of diagnoses since 2016 might represent less of an increase than currently believed. Instead, current rates might be a more accurate depiction of the burden of infection. Consideration of this point should guide future analyses of gonorrhea and chlamydia epidemiology.

\section{Conclusion}

We reviewed the diagnosis rates and numbers for gonorrhea and chlamydia pre/post-validation and implementation of extragenital NAAT and found that local increases in identified cases of these infections corresponded with the implementation of this new testing technology. Going forward, as extragenital NAAT for gonorrhea is adopted by more healthcare providers, it is possible that the observed rate of gonorrhea may continue to increase. Extragenital testing by NAAT among other non-gbMSM groups may further increase apparent rates-although further research is required to evaluate this. Eventually, with better testing and treatment, we might see a decrease in both the true and observed incidence of gonorrhea and possibly chlamydia as well. In the meantime, offering extragenital NAAT to gbMSM who engage in sexual practices involving extragenital sites with more than one sexual partner is good clinical and public health practice.

\section{Authors' statement}

DSF - Writing-original draft \& editing, conceptualization, statistical expertise POB - Writing-original draft \& editing, conceptualization, clinical expertise

\section{Competing interests}

None.

\section{Acknowledgements}

Both authors would like to thank $\mathrm{K}$ Cronin and $\mathrm{P}$ Nelson from Public Health Ontario Laboratory for data extraction.

POB would like to acknowledge his research chair in public health and HIV prevention from the Ontario HIV Treatment Network (OHTN). 


\section{Funding}

None.

\section{References}

1. Choudhri Y, Miller J, Sandhu J, Leon A, Aho J. Gonorrhea in Canada, 2010-2015. Can Commun Dis Rep 2018;44(2):37-42. DOI PubMed

2. Ontario Ministry of Health and Long-term Care (MOHLTC). integrated Public Health Information System (iPHIS) (accessed 2019-11-19). https://www.publichealthontario.ca/ en/diseases-and-conditions/infectious-diseases/ccm/iphis

3. Dewart CM, Bernstein KT, DeGroote NP, Romaguera R, Turner AN. Prevalence of rectal chlamydia and gonococcal infections: systematic review. Sex Transm Dis 2018;45(5):287-93. DOI PubMed

4. Lusk MJ, Uddin RN, Lahra MM, Garden FL, Kundu RL, Konecny P. Pharyngeal gonorrhea in women: an important reservoir for increasing Neisseria gonorrhoeae prevalence in urban Australian heterosexuals? J Sex Transm Dis 2013;2013:967471. DOI PubMed

5. Public Health Agency of Canada. Canadian Guidelines on Sexually Transmitted Infections. Ottawa (ON): PHAC (updated 2020-05-06). https://www.canada.ca/en/ public-health/services/infectious-diseases/sexual-healt h-sexually-transmitted-infections/canadian-guidelines/ sexually-transmitted-infections.html

6. Meyer T. Diagnostic procedures to detect Chlamydia trachomatis infections. Microorganisms 2016;4(3):25. DOI PubMed

7. Public Health Ontario. Chlamydia trachomatis and Neisseria gonorrhoeae - Implementation of Nucleic Acid Amplification Testing (NAAT) for Rectal and Pharyngeal Sites. Toronto (ON): PHO; 2019. https://www.publichealthontario. $\mathrm{ca} /-/$ media/documents/lab/lab-sd-005-chlamydia -gonorrhoeae-sensitivity-specificity.pdf?la=en

8. Chan PA, Robinette A, Montgomery M, Almonte A, Cu-Uvin S, Lonks JR, Chapin KC, Kojic EM, Hardy EJ. Extragenital infections caused by Chlamydia trachomatis and Neisseria gonorrhea: A review of the literature. Infect Dis Obstet Gynecol 2016;2016:5758387. DOI PubMed

9. Chamberlain N, Crosby RA, Mena L, Chan PA, Mayer KH. Is patient-reported exposure a reliable indicator of anogenital gonorrhea and chlamydia screening in young Black men who have sex with men (YBMSM)? Sex Transm Dis 2017;44(7):390-2. DOl PubMed

10. Andersson N, Boman J, Nylander E. Rectal chlamydia should screening be recommended in women? Int J STD AIDS 2017;28(5):476-9. DOI PubMed
11. United States Centers of Disease Control and Prevention. 2015 Sexually Transmitted Diseases Treatment Guidelines - Special Populations: MSM. Atlanta (GA): CDC (updated 2015-06-04; accessed 2020-03-28). https://www.cdc.gov/ std/tg2015/specialpops.htm\#MSM

12. den Heijer CD, van Liere GA, Hoebe CJ, van Bergen JE, Cals JW, Stals FS, Dukers-Muijrers NH. Who tests whom? A comprehensive overview of Chlamydia trachomatis test practices in a Dutch region among different STI care providers for urogenital, anorectal and oropharyngeal sites in young people: a cross-sectional study. Sex Transm Infect 2016 May;92(3):211-7. DOI PubMed

13. Lutz AR. Screening for asymptomatic extragenital gonorrhea and chlamydia in men who have sex with men: Significance, recommendations and options for overcoming barriers to testing. LGBT Health 2015;2(1):27-34. DOI PubMed

14. Tan DH, Hull MW, Yoong D, Tremblay C, O'Byrne $P_{1}$ Thomas R, Kille J, Baril JG, Cox J, Giguere P, Harris M, Hughes C, MacPherson P, O'Donnell S, Reimer J, Singh A, Barrett L, Bogoch I, Jollimore J, Lambert G, Lebouche B, Metz G, Rogers T, Shafran S; Biomedical HIV Prevention Working Group of the CIHR Canadian HIV Trials Network. Canadian guideline on HIV pre-exposure prophylaxis and nonoccupational postexposure prophylaxis. CMAJ 2017;189(47):E1448-58. DOI PubMed

15. Ward H, Rönn M. Contribution of sexually transmitted infections to the sexual transmission of HIV. Curr Opin HIV AIDS 2010;5(4):305-10. DOI PubMed

16. Barbee LA, Khosropour CM, Dombrowksi JC, Golden MR. New Human Immunodeficiency Virus diagnosis independently associated with rectal gonorrhea and chlamydia in men who have sex with men. Sex Transm Dis 2017;44(7):385-9. DOl PubMed

17. Bernstein KT, Marcus JL, Nieri G, Philip SS, Klausner JD. Rectal gonorrhea and chlamydia reinfection is associated with increased risk of HIV seroconversion. J Acquir Immune Defic Syndr 2010;53(4):537-43. DOI PubMed

18. Rosenberger JG, Dodge B, Van Der Pol B, Reece M, Herbenick D, Fortenberry JD. Reactions to self-sampling for ano-rectal sexually transmitted infections among men who have sex with men: a qualitative study. Arch Sex Behav 2011;40(2):281-8. DOI PubMed

19. Wayal S, Llewellyn C, Smith H, Hankins M, Phillips A, Richardson D, Fisher M; Home Sampling Kit Project Steering Group. Self-sampling for oropharyngeal and rectal specimens to screen for sexually transmitted infections: acceptability among men who have sex with men. Sex Transm Infect 2009;85(1):60-4. DOI PubMed

20. Rosenberger JG, Reece M, Schick V, Herbenick D, Novak DS, Van Der Pol B, Fortenberry JD. Sexual behaviors and situational characteristics of most recent male-partnered sexual event among gay and bisexually identified men in the United States. J Sex Med 2011;8(11):3040-50. DOI PubMed 
21. Brooks H, Llewellyn CD, Nadarzynski T, Pelloso FC, De Souza Guilherme F, Pollard A, Jones CJ. Sexual orientation disclosure in health care: a systematic review. $\mathrm{Br} \mathrm{J}$ Gen Pract 2018;68(668):e187-96. DOI PubMed
22. Coleman TA, Bauer GR, Pugh D, Aykroyd G, Powell L, Newman R. Sexual orientation disclosure in primary care settings by gay, bisexual, and other men who have sex with men. LGBT Health 2017;4(1):42-54. DOI PubMed

\title{
The Role of Preventive Medicine Technicians in protecting and promoting health in the Canadian Armed Forces
}

\author{
Master Warrant Officer Tonya Pugh ${ }^{1}$ \\ ${ }^{1}$ Directorate Force Health Protection, Canadian Forces Health Services, Ottawa, ON
}

Military personnel are deployed to a number of physically challenging/austere locations globally, which presents Canadian Armed Forces (CAF) personnel with some unique health hazards. Keeping CAF personnel healthy-both individually and as a population-are key objectives for the Preventive Medicine Technicians (PMed Tech) in supporting operational readiness (1).

Preventive Medicine Technicians are the strategic and tactical ground battlefield hygiene and sanitation inspectors in CAF. They represent Canadian Forces Health Services in the mitigation of non-battle-related illness, operating within the Directorate of Force Health Protection. The PMed Techs support the CAF healthcare system by performing hygiene, sanitation, environmental and occupational health inspections. They also collect and test water samples for potability and recreational use, manage integrated pest control procedures and provide guidance to deploying CAF members using recognized national and international health recommendations. Senior PMed Techs work collaboratively with the Epidemiology section to monitor and advise on communicable disease surveillance, such as vector, water and foodborne diseases, which can adversely affect CAF personnel and thus impact mission objectives. The PMed Techs routinely deploy internationally and domestically together with other CAF members in quite diverse environments (land, sea and air) to assist with health protection of military personnel. One example of their work is the mentorship training of foreign allies-for example, the Afghanistan Army, Air Force and Police-on the principals of disease and control measures. In summary, PMed Techs play an instrumental public health role in ensuring the health of CAF.

\section{Reference}

1. Schofield S, Tepper M, Campbell J. Health Protection of Armed Forces Personnel. In: Dawood R, editor. Travellers' Health: How to stay healthy abroad. 5th ed. Oxford UK: Oxford University Press; 2012, Chapter 11, Section 11.9:pg 419-426.

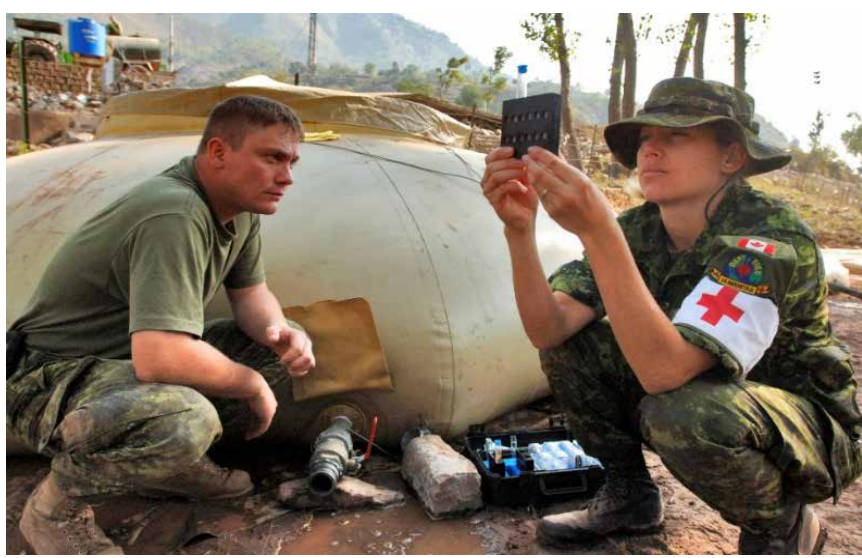

Canadian Armed Forces Preventive Medicine Technician testing water samples on deployment Photo credit: Canadian Armed Forces - Combat Camera 